\title{
Basosquamous Carcinoma: Report of Two Cases and Review of Literature
}

\author{
CH Saàdani ${ }^{1 *}$, H Baybay ${ }^{1}$, H ElMahi $^{1}$, KH Elboukhari ${ }^{1}$, S Gallouj $^{1}$, I SOUAF ${ }^{2}$ and FZ Mernissi ${ }^{1}$ \\ ${ }^{1}$ Departement of Dermatology, CHU Hassan II, Fes, Morocco \\ ${ }^{2}$ El Yousr laboratory, Fes, Morocco

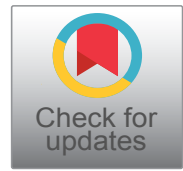 \\ *Corresponding author: CH Saàdani, Departement of Dermatology, CHU Hassan II, Fes, Morocco, E-mail: ch.saadani@ \\ gmail.com
}

\begin{abstract}
Background: Basosquamous cell carcinoma (BSCC) or metatypical carcinoma is a poorly known cutaneous tumor that is considered as an aggressive type of basal cell carcinoma with an increased risk of recurrences and metastases. The most common onset is in the head and neck region.

Methods: We present two cases of basosquamous carcinoma of lower extremity, in which a longstanding lesion initially diagnosed as basal cell carcinoma for one case, and as squamous cell carcinoma for the second case, and were later found to have basosquamous histology. Both patients received surgical treatment with good evolution.

Conclusion: Metatypical basal cell carcinoma or basosquamous Cell carcinoma (BSCC), is a rare skin carcinoma, potentially aggressive entity with clinical and pathological characteristics of $\mathrm{CBC}$ and $\mathrm{CSC}$, considered as a variant of $\mathrm{CBC}$, but is currently defined as a tumor complex. Although clinically similar to $\mathrm{BCC}$, it is more aggressive and metastasizes more frequently. Clinical differentiation of BSC and $B C C$ is impossible owing to their clinical similarity. Dermoscopic patterns may facilitate early diagnosis and accurate management. Confirmation remains histological, showing areas typical for both BCC and SCC. Immunohistochemical examination may provide additional data for a more accurate diagnosis. Review of the literature reveals a metastatic rate greater than that of basal cell and squamous cell carcinoma, and identifies several important characteristics that impact prognosis after surgical resection. It's important to have an early diagnosis and compulsory excision and a regular follow-up in order to avoid the significant morbidity and even mortality associated with this aggressive carcinoma of the skin.
\end{abstract}

\section{Keywords}

Basosquamous, Carcinoma, Metastasis

\author{
Abbreviation \\ BSCC: Basosquamous Cell Carcinoma; BCC: Basal Cell \\ Carcinoma; SCC: Squamous Cell Carcinoma
}

\section{Introduction}

Basosquamous cell carcinoma (BSCC) or metatypical carcinoma, is a rare type of epithelial skin tumor first described by MacCormac in 1910, regarded by most authors as a rare variant or subtype of basal cell carcinoma (BCC) [1], it has areas of definitive basal cell carcinoma and squamous cell carcinoma with a transition zone between them, suggesting differentiation of one of the tumours into the other.

BSC is a rare tumor with an incidence of less than $2 \%$ of all non-melanoma skin cancers [2] and is predominant in men. Its etiology is multifactorial, but UV radiation, aging, and tobacco exposure seem to play key roles in the onset of BSC [3]. BSCs are usually located in the head and neck area or in other sun-exposed areas [3].

Although basosquamous carcinoma is similar to the basal cell carcinoma clinically and morphologically, this tumour may behave more aggressively, with a propensity for local recurrence and a potential risk for distant metastatic spread. However, there is a disagreement about aggressiveness of this tumour, and data are insufficient [2].

\section{Case Report 1}

A 59-year-old man, with past medical history was 


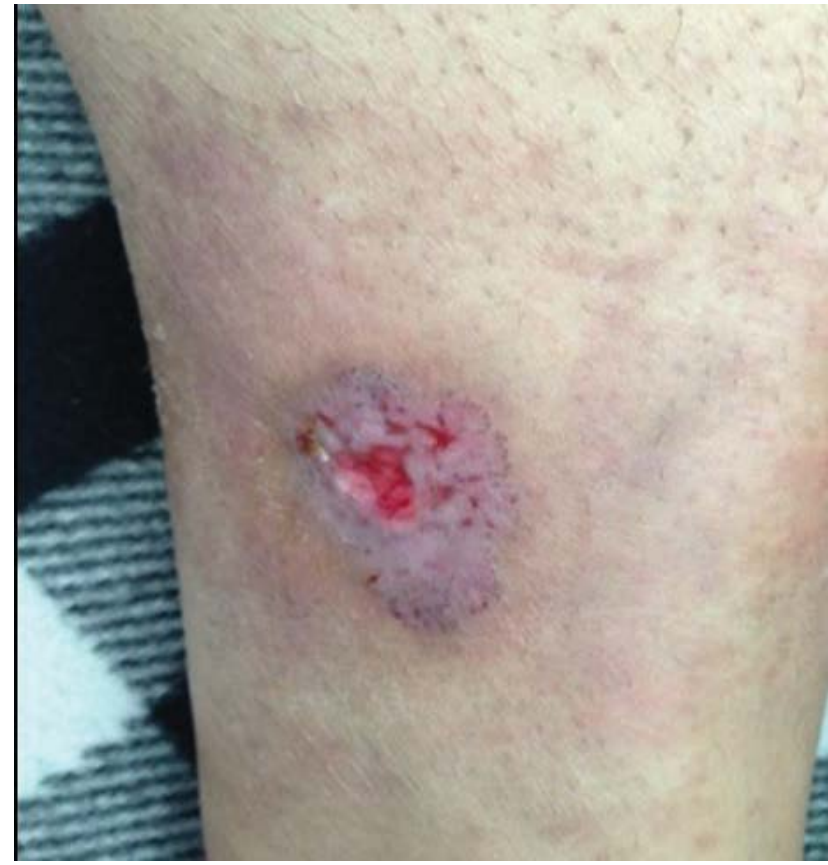

Figure 1: Clinical image: Ulceration in the leg (case 1).

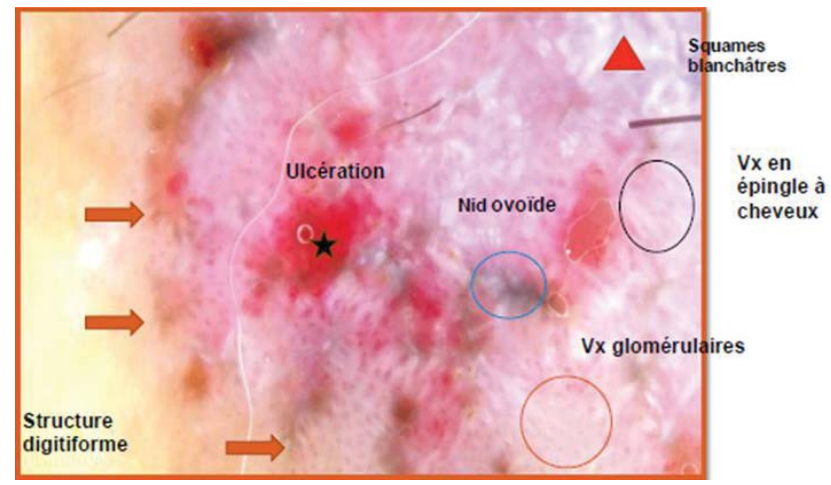

Figure 2: Dermoscopic features (case 1).

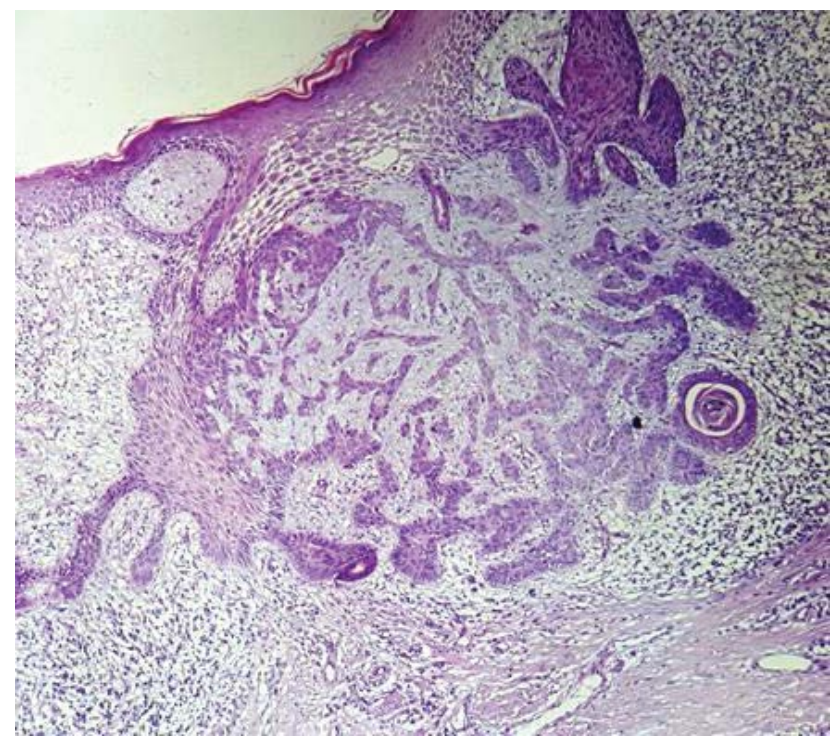

Figure 3: Histological section of basal cell carcinoma (case 1).

significant for diabetes, and hypertension, presented a painless, slightly pruriginous left leg injury, evolving for 10 months, Whose diagnosis of squamous cell carcino-

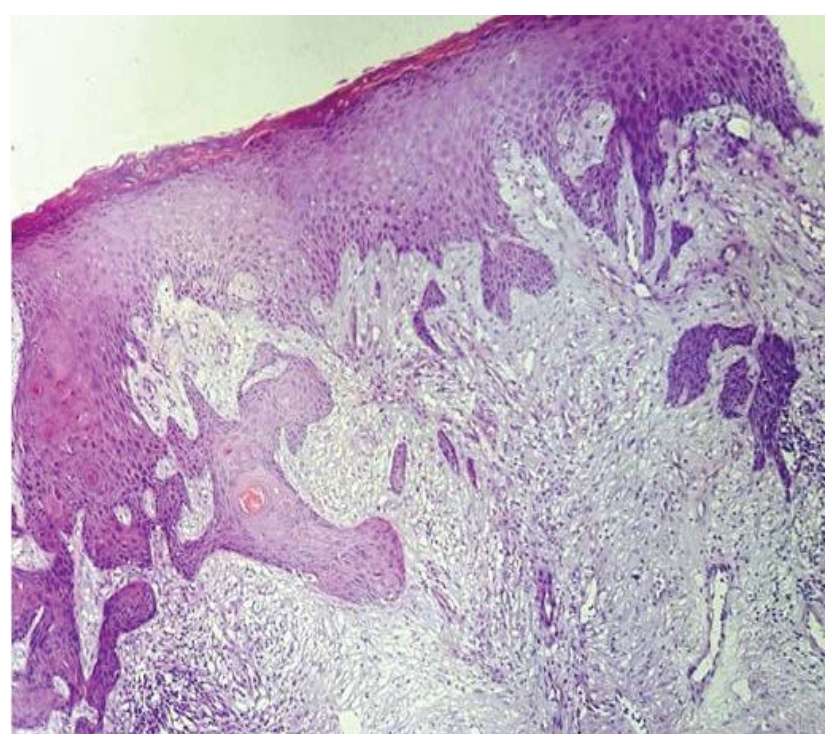

Figure 4: Histological section of basal cell carcinoma (case 1).

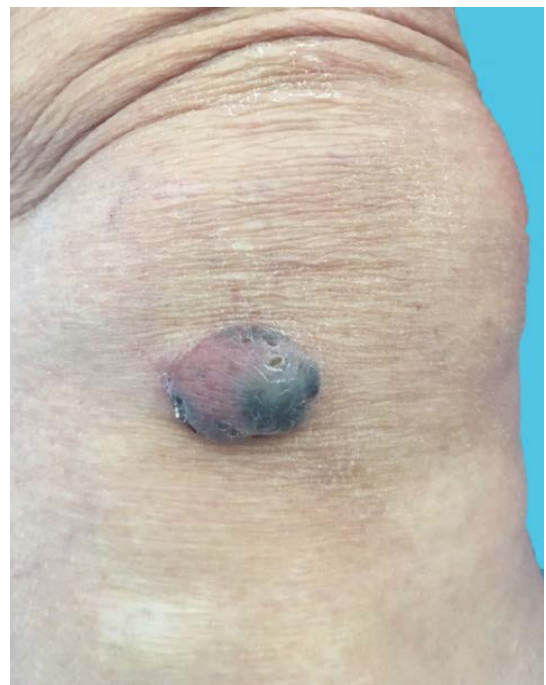

Figure 5: Image Clinical image: Nodule at the knee (case 2).

ma (SCC) was histologically confirmed by a biopsy. Clinical examination revealed a large ulcerated skin lesion, 3 $\mathrm{cm}$ in size, well limited, with clean surface, covered with whitish scales in places resting on a slightly atrophic skin (Figure 1). Dermoscopy focuses on diagnosis of squamous cell carcinoma by demonstrating glomerular and hairpins vascularity surrounded by a whitish halo (Figure 2). Other lesions were found, including ovoid nests, ulceration and digitiform structures, suggestive of the diagnosis of associated basal cell carcinoma (Figure 2). Histological examination of the surgical specimen with a margin of $10 \mathrm{~mm}$ revealed basosquamous carcinoma with disease-free resection margins (Figure 3 and Figure 4). The extension rate was negative. Currently, after 1 year, the patient is free of disease and enjoys good health.

\section{Case Report 2}

A 68-year-old woman, Followed for idiopathic thrombocytopenic purpura, presented an asymptomatic nodular lesion, on the left knee, evolving for 1 year. 


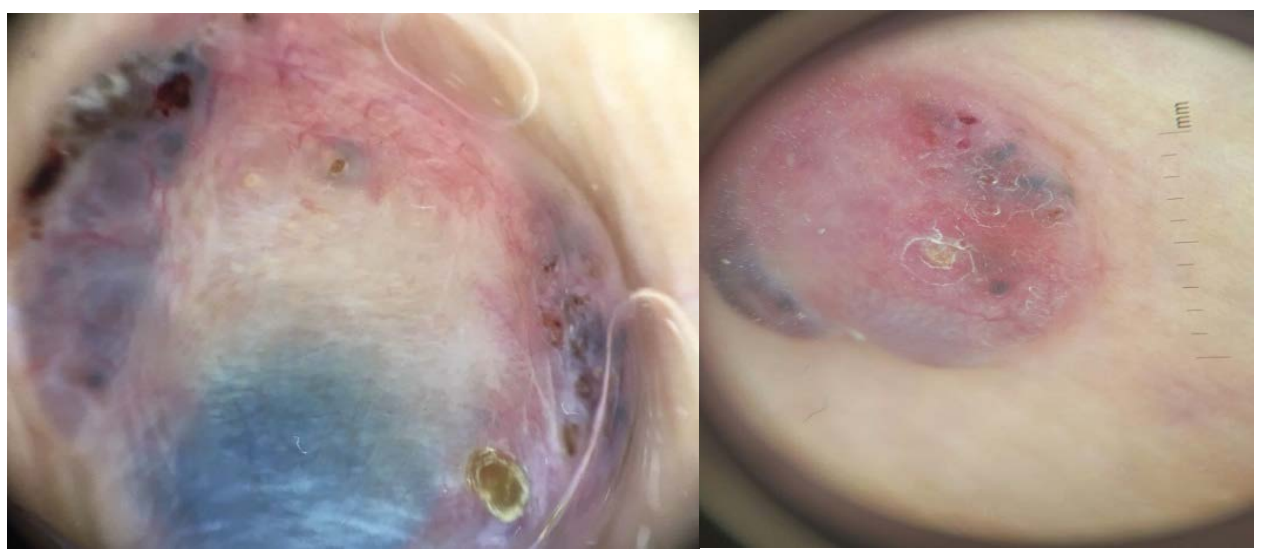

Figure 6: Dermoscopic features (case 2).

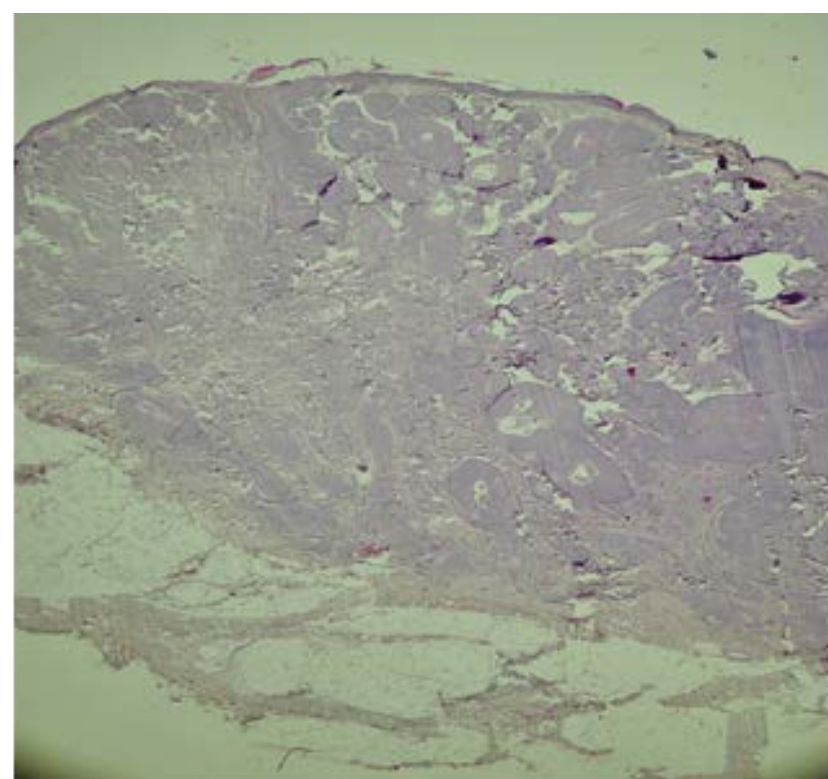

Figure 7: Histological section of basal cell carcinoma (case 2).

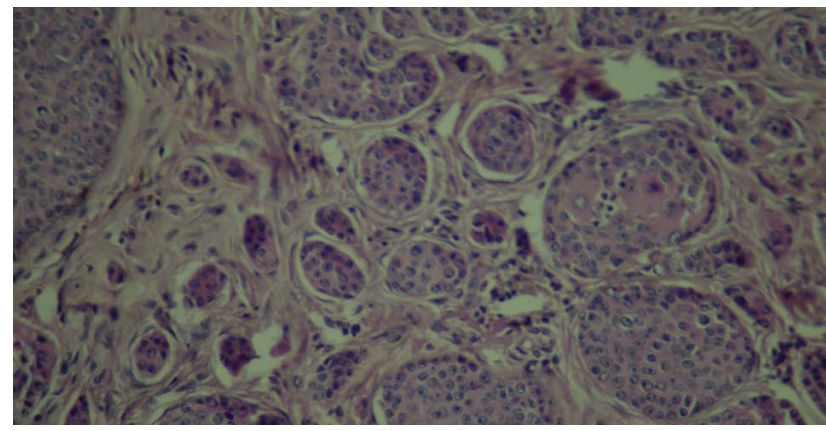

Figure 8: Histological section of basal cell carcinoma (case 2)

Clinical examination established a nodule, $2 \mathrm{~cm}$ in size, well limited, covered with telangiectasia (Figure 5). Dermoscopy confirmed the diagnosis of basal cell carcinoma by demonstrating ovoid nests, digitiform structures, and télangiectasia (Figure 6). Staging procedures did not reveal any evidence of metastatic disease. Histological examination of the surgical specimen with a margin of $6 \mathrm{~mm}$, revealed an infiltrating tumour with a mixed basal and spindle cell pattern with disease-free resection margins (Figure 7 and Figure 8). Immunohistochemi-

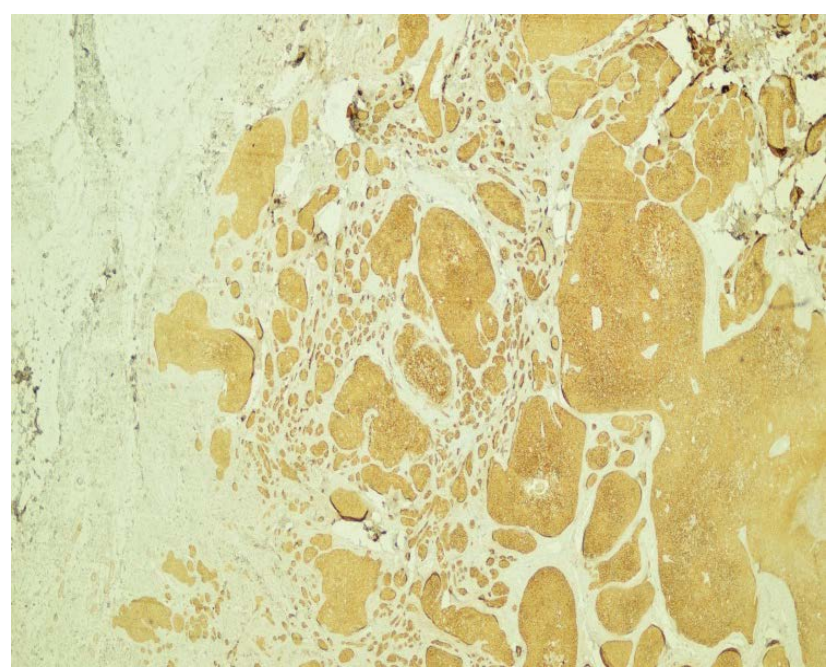

Figure 9: Histological section of basal cell carcinoma and IHC (CK 5/6) (case 2).

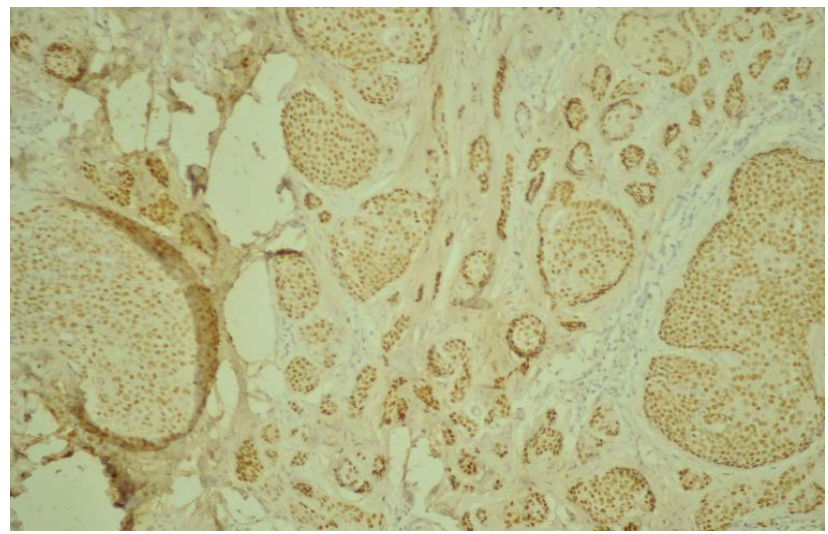

Figure 10: Histological section of basal cell carcinoma and IHC (P63) (case 2).

cal (IHC) analysis with p63 and CK5/6 demonstrated a mixed pattern of staining consistent with a diagnosis of a basosquamous carcinoma (BSC Figure 9 and Figure 10). The extension rate was negative. Currently, after 1 year, the patient is free of disease and enjoys good health.

In both cases, IHC was performed on the sections, using a complete panel of specific antibodies. BSCs showed 
a transition zone between typical BCC and SCC and staining patterns similar to both the BCC and SCC. The lesions stained positive for cytokeratins $5 / 6$ and $p 63$ in squamoid areas (Figure); and for EMA in classic areas of BCC.

\section{Discussion}

Metatypical basal cell carcinoma (MTBCC), also known as basosquamous carcinoma (BSC), is a rare skin carcinoma [4]. It was first described in 1910 by MacCormac and later, in 1928, by Montgomery, using the terms "metatypical basal cell carcinoma" or "basal squamous cell epithelioma" as synonyms of BSC, a pathological entity described as transitional between BCC and SCC [5]. In 1959, Burston and Clay defined it as a variant of BCC, differentiating into SCC. This hypothesis of "squamatization" of BCC was cytologically demonstrated by the presence of areas of basal cells differentiating into squamous cells [5].

The incidence of BSC is ill-defined; it may represents $1.2 \%$ to $2.7 \%$ of all skin carcinomas [6]. Although clinically similar to BCC, it is more aggressive and metastasizes more frequently (> 7.4\%), even in comparison to SCC (0.78\%) [4]. Metastases have been described in the lymph nodes, lung and bones, and appear 4-7 years after presentation of the primary tumor [7].

Some common characteristics have emerged when many patients with BSC are compared [6]. In large series, $\mathrm{BSC}$ is most commonly found in the head and neck region, and less common in the trunk, limbs (as reported in our two cases), scalp and other regions. Additionally, there is a male predominance and a mean age distribution in the $7^{\text {th }}$ decade of life [6]. Recurrences occurred mainly in the head and neck area [2].

Clinical differentiation of BSC and BCC is impossible owing to their clinical similarity [8]. Dermoscopic patterns may facilitate early diagnosis and accurate management, which comprises BCC-related criteria, as well as features that are known to characterize invasive SCC [9]. The most frequently detected criteria were: Unfocused (peripheral) arborizing vessels keratin masses, white structureless areas, superficial scale, ulceration or blood crusts, white structures, blue-grey blotches and blood spots in keratin masses $[9,10]$. BSC appears to have overlapping dermoscopic features of BCC and invasive SCC, and detection of at least one dermoscopic criterion of both BCC and SCC should raise suspicion for the tumor [10].

Therefore, histopathological examination is mandatory before primary treatment [8]. Histologically BSC is classified in two type-intermediate and mixed [8]. The first one shows areas typical for both BCC and SCC, transitional zones and tumor islets found together. In the mixed type, typical basal cells coexist with areas of squamous cells and possibly squamous pearls. A large number of mitoses are usually found [8].

Immunohistochemical examination (where avail- able) may provide additional data for a more accurate diagnosis, typically showing strong positivity for keratins 17 and 8 in BCC, and 6 to 8 times decreased intensity of staining in BSC [8].

Another point of distinction between $\mathrm{BCC}$ and $\mathrm{BSC}$ is mitotic activity: $B S C$ is more proliferative, with a mitotic activity that is eight times greater that of the normal epidermis, compared with BCC in which it is only four times greater. In addition, BSC also shows a more frequent presence of atypical mitotic figures, such as multipolar or tripolar mitoses [8].

In light of the rarity and the complex diagnosis of BSC, it is often not clear enough whether some clinical or histological features might be correlated with the risk of metastasis and/or recurrence [5]. This aggressive course may be attributed to different factors: Male gender, patient immune response, with weak or absent inflammatory host response and/or the local sclerotic tissue reaction, tumor dimension, anaplasia degree of the SCC component, which is responsible for the metastatic potential, since the $\mathrm{BCC}$ rarely metastasizes [5]. Also the presence of positive resection margins, lymphatic invasion, and especially perineural invasion (PNI) seem to be significantly correlated to higher rates of metastatic disease and/or recurrence.

Early and adequate surgical treatment of the primary BSC is mandatory. Although the tumor resection is performed with large safety margins for well-known infiltrative growth pattern of this tumor and even though most surgeons decide the size of the safe surgical margin according to a dermoscopic presurgical evaluation of tumor margins or by the use of MMS, local recurrence rates are significantly high, ranging between $12.1 \%$ and $45.7 \%$ [5]. Radiotherapy is an additional option for cases with high risk of relapses (positive excision margins, deep invasion, $\mathrm{pN}+$, and perineural/intravascular spread). The role of chemotherapy is not established. In case of lymphnode metastasis, lymphatic mapping and sentinel lymph node biopsy (a standard approach in malignant melanoma) can also be performed, especially in high risk lesions. A regular follow-up would allow early detection of disease recurrence [6].

\section{Conclusions}

Although uncommon among skin carcinomas, BSC commands special attention for its diagnostic challenges and metastatic potential. Our cases highlight the need to consider this rare and aggressive variant of BCC and the role of dermoscopy in suspecting this variety. The location at the limb, far from the cephalic region, considers the low risk of metastasis and recurrences. However, It is vital to appreciate the importance of early resection with free margins, a full workup for lymph node and distant metastases, and a regular follow up to detect a disease recurrence. 


\section{Declaration of Conflicting Interests}

The author(s) declared no potential conflicts of interest with respect to the research, authorship, and/or publication of this article.

\section{References}

1. Mougel F, Kanitakis J, Faure M, Euvrard S (2012) Basosquamous cell carcinoma in organ transplant patients: $A$ clinicopathologic study. J Am Acad Dermatol 66: e151-e157.

2. Kececi Y, Argon A, Kebat T, Sir E, Gungor M, et al. (2015) Basosquamous carcinoma: Is it an aggressive tumor. $J$ Plast Surg Hand Surg 49: 107-111.

3. Wermker K, Roknic N, Goessling K, Klein M, Schulze HJ, et al. (2015) Basosquamous Carcinoma of the Head and Neck: Clinical and Histologic Characteristics and Their Impact on Disease Progression. Neoplasia 17: 301-305.

4. Garcia C, Poletti E, Crowson AN (2009) Basosquamous carcinoma. J Am Acad Dermatol 60: 137-143.

5. Bucci T, Santoro A, Pannone G, Rodriguez J, Fior A, et al. (2016) Metastatic Basosquamous Carcinoma: Report of
Two Cases and Clinicopathological Considerations. Int J Surg Pathol 24: 726-732.

6. Costantino D, Lowe L, Brown DL (2006) Basosquamous carcinoma-an under-recognized, high-risk cutaneous neoplasm: Case study andreview of the literature. J Plast Reconstr Aesthet Surg 59: 424-428.

7. H Dhouib, M Mnejja, L Ayadi, B Hammami, T Boudawara, et al. (2009) Carcinome basocellulaire metatypique. Anna d'Otolaryngologie et de Chirurgie Cervico-faciale 126: 25-28.

8. Tchernev G, Chokoeva AA, Penev PK, Ananiev J, Nowak A, et al. (2014) Relapsing advanced metatypical basal cell carcinomas (MTBCC) of the face: Surgical modalities. Wien Med Wochenschr 164: 529-535.

9. Hakima Elmahi, Asmae Lahlou, Hanane Baybay, Salim Gallouj, Fatima Zahra Mernissi, et al. (2016) L'apport de la dermoscopie dans le carcinome basosquameux. Pan Afr Med J 25: 252.

10. Giacomel J, Lallas A, Argenziano G, Reggiani C, Piana S, et al. (2013) Dermoscopy of basosquamous carcinoma. $\mathrm{Br}$ J Dermatol 169: 358-364. 